\title{
Area-Selective Growth of Aligned ZnO Nanorod Arrays for MEMS Device Applications ${ }^{+}$
}

\author{
Jiushuai Xu *, Klass Strempel, Hao Zhou, Andreas Waag, Maik Bertke, Angelika Schmidt \\ and Erwin Peiner
}

TU Braunschweig, Department of Semiconductor Technology, Braunschweig, Germany and Laboratory for Emerging Nanometrology (LENA), 38106, Braunschweig, Germany; k.strempel@tu-bs.de (K.S.); hao.zhou@tu-bs.de (H.Z.); a.waag@tu-bs.de (A.W.); m.bertke@tu-bs.de (M.B.); angelika.schmidt@tu-bs.de (A.S.); e.peiner@tu-bs.de (E.P.)

* Correspondence: jiushuai.xu@tu-bs.de; Tel.: +49-531-391-3806

+ Presented at the Eurosensors 2018 Conference, Graz, Austria, 9-12 September 2018.

Published: 23 November 2018

\begin{abstract}
ZnO}$ nanorods (NRs) arrays with good vertical alignment were selectively grown on microscale patterned surfaces by a MEMS-compatible, low-temperature chemical-bath deposition method (CBD). The direct-current (DC) sputtered and subsequently annealed $\mathrm{ZnO}$ seed-layer was found to have a crucial effect on the $\mathrm{ZnO}$ NRs growth. Depending on the preannealing temperature between $200{ }^{\circ} \mathrm{C}$ and $700{ }^{\circ} \mathrm{C}$, which is compatible with our microcantilever fabrication process, diameters and area densities of the NRs of 60-99 nm and $17-27 \mu \mathrm{m}^{-2}$ were observed, respectively, with the best alignment at $600{ }^{\circ} \mathrm{C}$. A surface-area enlargement factor of 48 was achieved with respect to a $\mathrm{ZnO}$ layer indicating the potential of $\mathrm{ZnO}$ NRs arrays for MEMS applications, such as gas sensing.
\end{abstract}

Keywords: area-selective growth; $\mathrm{ZnO}$ nanorods; MEMS; DC sputtering/annealing

\section{Introduction}

In recent years, one-dimensional (1D) $\mathrm{ZnO}$ nanostructures have received great interest due to their potential applications in electronic and optoelectronic devices, such as solar cells, gas sensors, photodetectors, light-emitting diodes and surface acoustic wave devices. Various methods have been studied to fabricate aligned $1 \mathrm{D} \mathrm{ZnO}$ nanostructures, including thermal vacuum evaporation (TVE), electron beam gun evaporation, molecular beam epitaxy (MBE), metal-organic chemical vapor deposition (MOCVD), sputtering techniques, chemical bath deposition (CBD), etc. [1].

However, for the moment, it is still challenging to achieve the growth of functional $\mathrm{ZnO}$ nanostructures with highly aligned and oriented arrays on micro and nanoscale surfaces, which are crucial for the performance of these devices. The low-temperature CBD method is a simple facility, no-catalyst and low-cost process [2]. However, before the growth of ZnO NRs, an aqueous spin-coating method or sol-gel alkaline solution is essential for seed-layer (SL) coating, which are not compatible with MEMS fabrication [3]. In this paper, we report a CBD-based twosteps process, by using a DC sputtering/annealing (S/A) method for SL deposition. The areaselective growth of aligned $\mathrm{ZnO}$ nanorods (NRs) arrays was achieved on n-type silicon microcantilevers, indicating its applicability for MEMS device fabrication. In comparison with a sol-gel seed-layer deposition method, the DC-sputtered $\mathrm{ZnO}$ seed-layer has some advantages, such as easy thickness control, good morphology and high process repeatability. Compared with radio frequency (RF) magnetron-sputtering, which has a base pressure about $9 \times 10^{-5} \mathrm{~Pa}$ and a 
working Ar pressure of $5 \times 10^{-3} \mathrm{~Pa}$ [2], DC-sputtering can be operated at a moderate working pressure of $640 \mathrm{~Pa}$, and has a much lower power consumption. Furthermore, it has been found that the properties of $\mathrm{ZnO}$ NRs have a dependence on the pre-annealing of the seed-layer either by sol-gel deposition or by RF magnetron sputtering, so in this paper, DC-sputtered Zinc films were annealed under different temperatures to prepare seed-layers for subsequent $\mathrm{ZnO} \mathrm{NRs}$ growth by CBD. We find that the crystallinity, resistivity and morphology of the ZnO NRs strongly depend on the pre-annealing of the seed-layers.

\section{Experimental}

Area-Selective Growth of Aligned $\mathrm{ZnO}$ Nanorods Arrays

Figure 1 depicts the fabrication process steps of area-selective growth of aligned $\mathrm{ZnO}$ nanorods (NRs) arrays on $n$-type silicon, and the details are described as follows:

(a) The fabrication started from a piece of sample with a dimension of $30 \times 30 \mathrm{~mm}^{2}$, the sample was cut from an $n$-type bulk-silicon wafer (crystal orientation: $<100>$; resistivity: $1-10 \Omega \times$ $\mathrm{cm}$; thickness: $275 \pm 15 \mu \mathrm{m}$ and diameter: $100 \pm 0.13 \mathrm{~mm}$ ) and cleaned by putting the sample into a boiling acid mixture $\left(\mathrm{H}_{2} \mathrm{O}_{2}(30 \%)\right.$ and $\left.\mathrm{H}_{2} \mathrm{SO}_{4}(96 \%), v: v=1: 1\right)$ for $5 \mathrm{~min}$.

(b) A positive photoresist (AZ 5214E, Merck, Kenilworth, NJ, USA) was utilized during the subsequent photolithography step and a MJB4 mask aligner (SÜSS MicroTec AG, Garching, Germany) was used to expose the pattern area. Prior to the exposure, the photoresist spincoating procedure was run at a speed of $5000 \mathrm{rpm}$ for $35 \mathrm{~s}$, to create a homogenous photoresist layer of $1.5 \mu \mathrm{m}$ in thickness. After the exposure, the sample was then dipped and developed in AZ 726 MIF developer solution (MicroChemicals, Merck) for $60 \mathrm{~s}$, followed by DI water rinsing and nitrogen purging.

(c) Afterwards, a polycrystalline Zn film was prepared by sputtering Zn (99.99\%) using high purity $\operatorname{Ar}(99.99 \%)$ gas under $50 \mu \mathrm{A}$ direct current $(\mathrm{DC})$, at room temperature $\left(25^{\circ} \mathrm{C}\right)$ and a working pressure of $640 \mathrm{~Pa}$. To obtain the selective deposition of sputtering film on the patterned area, the excess $\mathrm{ZnO}$ was removed using photoresist lift-off.

(d) Then the sputtered $\mathrm{Zn}$-film was annealed in an oven with open atmosphere, to investigate influence of annealing temperature on the nanorods growth, the sputtered samples were annealed at $200{ }^{\circ} \mathrm{C}, 300{ }^{\circ} \mathrm{C}, 400{ }^{\circ} \mathrm{C}, 500{ }^{\circ} \mathrm{C}, 600^{\circ} \mathrm{C}$ and $700{ }^{\circ} \mathrm{C}$, respectively.

(e) When the seed-layer has been prepared, a subsequent photolithography step was implemented corresponding to step (b) to protect the substrate during the next CBD process.

(f) ZONRs were grown by dipping the sample in an aqueous solution, which consisted of 30 $\mathrm{mmol} / \mathrm{L}$ zinc nitrate $\left(\mathrm{Zn}\left(\mathrm{NO}_{3}\right)_{2}\right)$ and $30 \mathrm{mmol} / \mathrm{L}$ hexamethyleneteramine ( $\left.\mathrm{HMT}, \mathrm{C}_{6} \mathrm{H}_{12} \mathrm{~N}_{4}\right)$, respectively. The deposition was carried out in a temperature-controlled chemical reactor, which was additionally equipped with a thermometer and a reflux condenser, for $3 \mathrm{~h}$ at $90{ }^{\circ} \mathrm{C}$. After the reaction, the sample was cleaned with acetone and deionized water, successively [4].

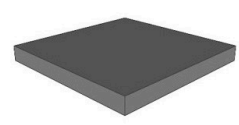

(a) n-type Si

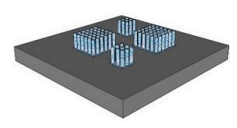

(f) $\mathrm{CBD}$
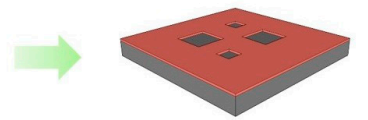

(b) Photolithography

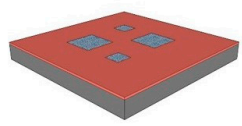

(e) Photolithography

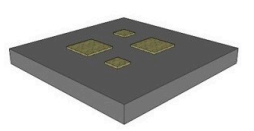

(c) Zn Sputter-coating

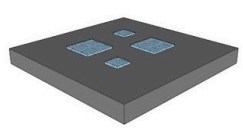

(d) Pre-annealing

Figure 1. Schematic diagram of area-selective growth of aligned $\mathrm{ZnO}$ nanorods (NRs) on $n$-type silicon. 


\section{Results and Discussion}

Figure 2 shows the top-view SEM graphs with a tilt angle of $30^{\circ}$ of the $\mathrm{ZnO}$ NRs grown on the $\mathrm{ZnO}$ seed-layers annealed at different temperatures, all the NRs were grown in the chemical bath at $90{ }^{\circ} \mathrm{C}$ for $3 \mathrm{~h}$. As it can be seen from the graphs, the obtained ZnO NRs were vertically oriented with respect to the substrates, the NRs based on the seed-layers annealed at $600{ }^{\circ} \mathrm{C}$ (NRs-600) tends to have the best orientation.

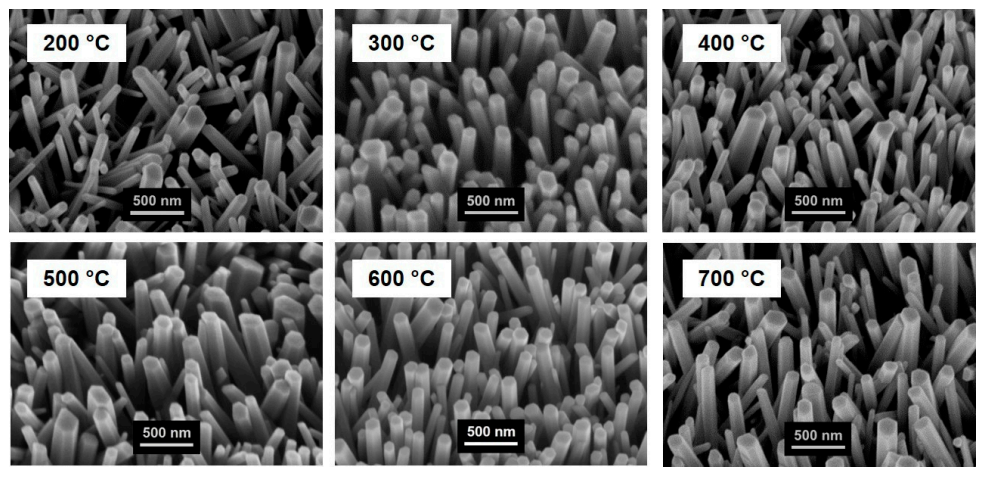

Figure 2. Inclined-view $\left(30^{\circ}\right) \mathrm{SEM}$ graphs of $\mathrm{ZnO}$ NRs grown on S/A SLs at different preannealing temperatures from $200{ }^{\circ} \mathrm{C}$ to $700{ }^{\circ} \mathrm{C}$.

The density and average diameters of the corresponding ZnO NRs on the seed-layers annealed at different temperatures are calculated according to Figure 2 and listed as Table 1. NRs-600 arrays possessing highest density and a relative big diameter, which means high surface-to-volume-ratio and that is important to the improve MEMS devices performance.

Table 1. Summary of the characterized parameters of NRs grown at different pre-annealing temperature.

\begin{tabular}{ccccccc}
\hline & $200{ }^{\circ} \mathrm{C}$ & $300{ }^{\circ} \mathrm{C}$ & $400{ }^{\circ} \mathrm{C}$ & $500{ }^{\circ} \mathrm{C}$ & $600{ }^{\circ} \mathrm{C}$ & $700{ }^{\circ} \mathrm{C}$ \\
\hline Diameter $(\mathbf{n m})$ & $60 \pm 20$ & $87 \pm 27$ & $66 \pm 24$ & $99 \pm 31$ & $93 \pm 24$ & $74 \pm 26$ \\
\hline Area density $\left(\boldsymbol{\mu m}^{-2}\right)$ & $25 \pm 5$ & $21 \pm 1$ & $27 \pm 2$ & $21 \pm 5$ & $27 \pm 2$ & $17 \pm 4$ \\
\hline
\end{tabular}

XRD was further used to characterize the crystallinity of different NRs arrays. As shown in Figure 3, the indexed diffraction peaks are consistent with the standard values of the bulk $\mathrm{ZnO}$ crystal (JCPDS 36-1451) and all the ZnO NRs have a wurtzite structure. The sharp and strong (002) peak indicates that the $\mathrm{ZnO}$ NRs have a potential c-axis orientation on sputtered/annealed ZnO SLs. Besides, we did not observe the (100) and (101) peaks which can be found in some samples grown on the sol-gel seed-layers [5]. The (002) diffraction peak of NRs-600 has the highest intensity, showing that NRs-600 arrays have the highest (002) c-axis orientation preference, revealing the best NRs vertical alignment as Figure 2. Furthermore, the resistivity of $\mathrm{ZnO}$ NRs arrays was measured using a four-point probe, and the results are depicted in Figure 4. The observed highest resistivity of the NRs-600 was thought to be caused by their best vertical alignment as visible in Figures 2 and 3.

We reported the fabrication and humidity sensing performance of $\mathrm{ZnO}-\mathrm{NRs}$-patterned piezoresistive silicon MEMS microcantilevers [6], based on the aforementioned two-step deposition method. In the present study, ZnO NRs were coated on the back surface of the microcantilevers, a schematic and SEM graphs of a microcantilever coated with NRs of $6 \mu \mathrm{m}$ in length solely on its back surface was displayed in Figures 5 and 6, respectively. A surfacearea enlargement factor of 48 was found, indicating its considerable application potential for MEMS devices. 


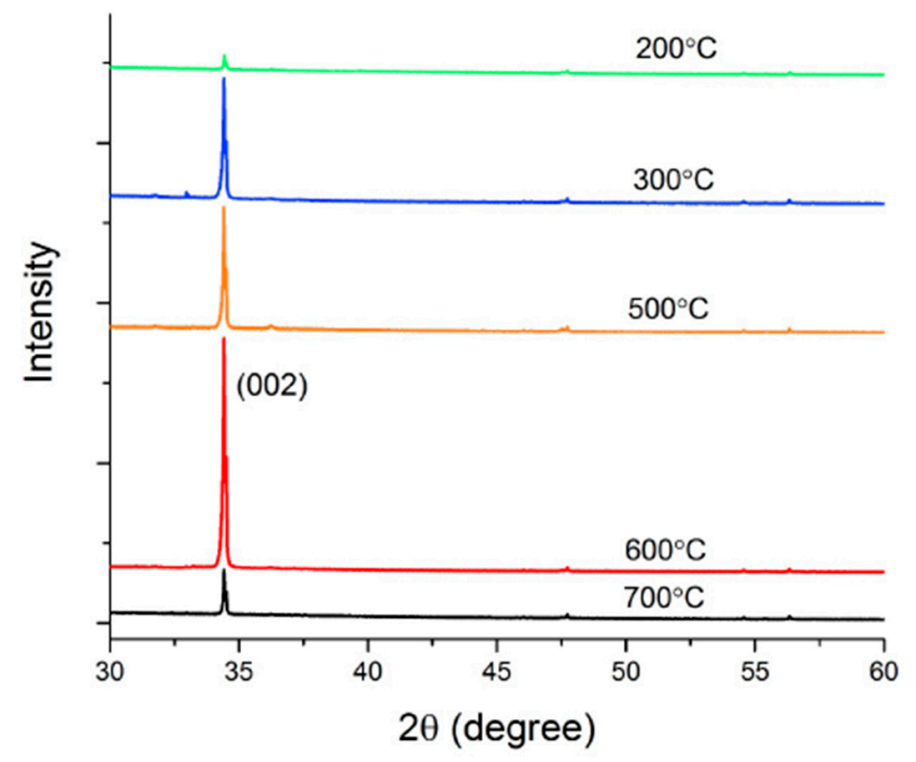

Figure 3. XRD spectra of ZnO NRs grown on SLs pre-annealed at different temperatures.

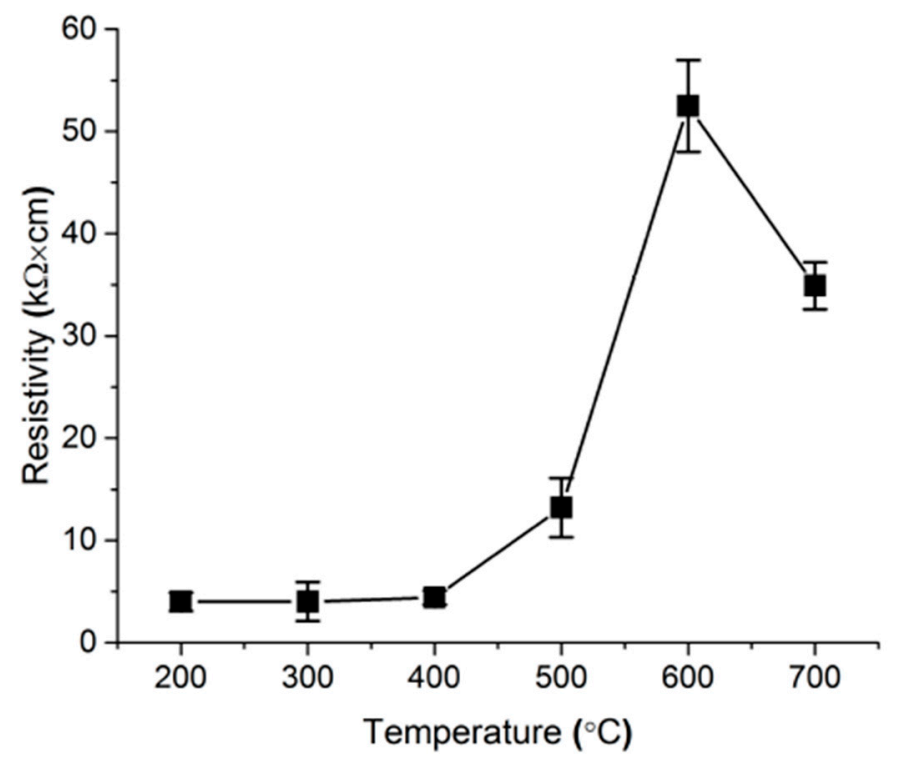

Figure 4. Resistivity of ZnO NRs array grown on a SL annealed at different temperatures.

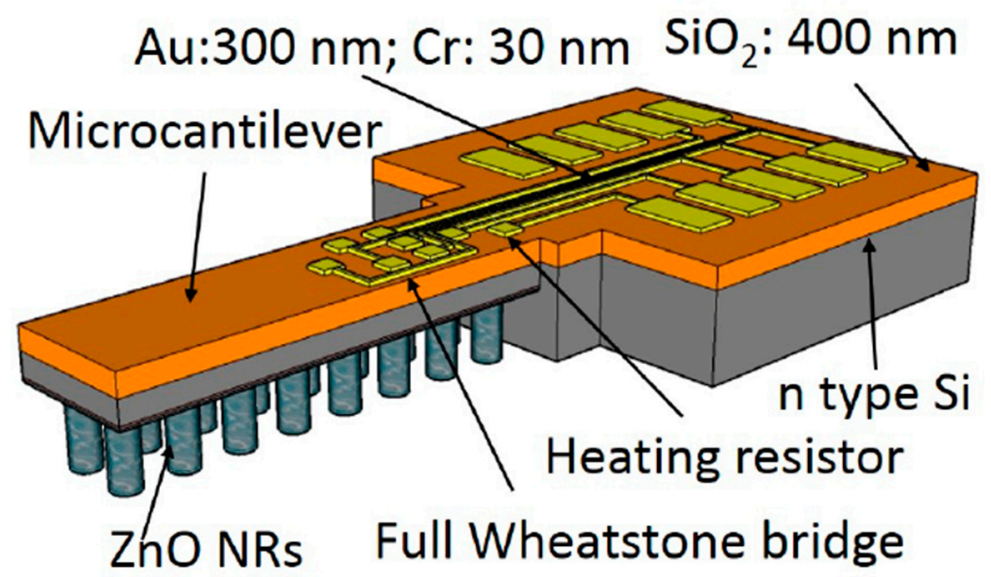

Figure 5. Schematic graph of a silicon microcantilever patterned with $\mathrm{ZnO}$ NRs on its back surface. 


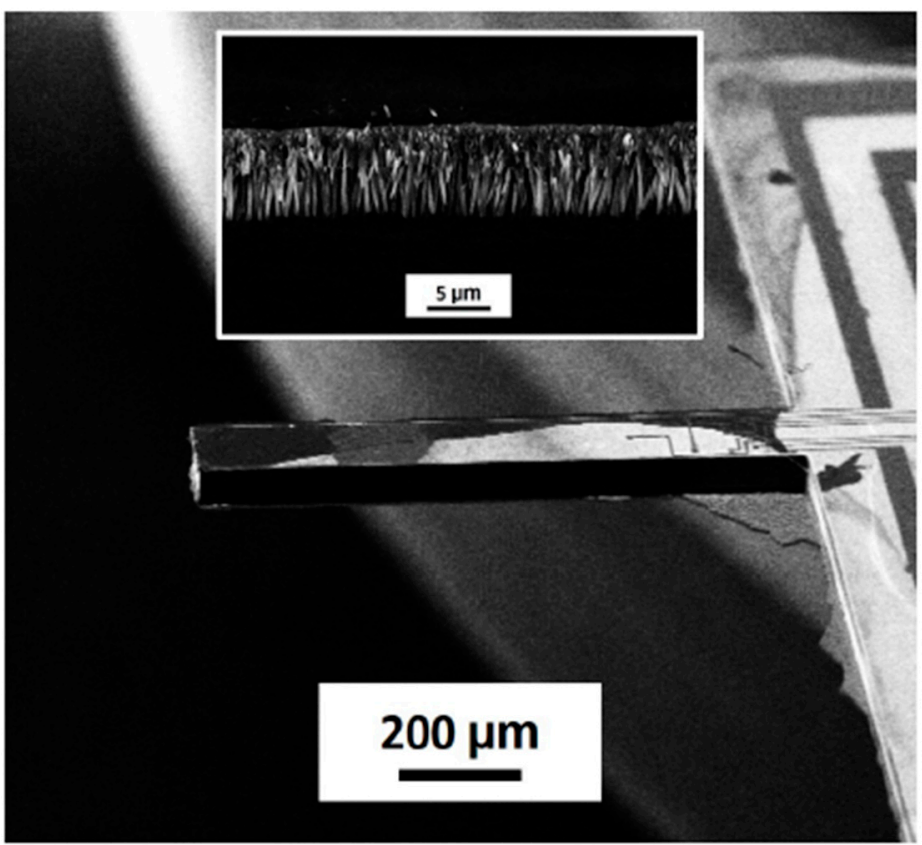

Figure 6. Inclined-view SEM graph of a Si microcantilever with ZnO NRs grown on its back surface (inset).

\section{Conclusions}

In this work, a DC-sputtered/annealed $\mathrm{ZnO}$ seed-layer and chemical-bath deposition based two-step $\mathrm{ZnO}$ NRs arrays growth method was introduced, and $\mathrm{ZnO}$ NRs arrays grown on seed-layers annealed under different temperatures were characterized to study their area density, diameter, crystallinity and resistivity. The SEM graphs, XRD patterns and four-point probe resistivity measurements illustrate that NRs arrays grown on the seed-layer annealed at $600{ }^{\circ} \mathrm{C}$ have the best $c$-axis orientation and vertical alignment as well as the highest surfacearea enlargement factor of 48 (with $6 \mu \mathrm{m}$ in length). Next, further properties relevant for MEMS devices applications, e.g., growth of NRs on different materials, vacancy concentrations and optical properties will beinvestigated.

Author Contributions: J.X. and E.P. conceived and designed the experiments; J.X. and A.S. performed the experiments; K.S., H.Z. and M.B. analyzed the data; A.W. and E.P. contributed reagents/materials/analysis tools; J.X. wrote the paper, E.P. revised the paper, A.W. and E.P. supervised the research.

Acknowledgments: Juliane Breitfelder, and Karl-Heinz Lachmund provided valuable technical support, J.X. is grateful for funding by the China Scholarship Council (CSC) under the Grant CSC No. 201506300019. Furthermore, we acknowledge the support from "Niedersachsisches Vorab", Germany, through the "Quantum- and anometrology (QUANOMET)" initiative within the project of "NP 2-2 and from the German research Foundation (DFG) under no. PE 885/3-1.

Conflicts of Interest: The authors declare no conflict of interest.

\section{References}

1. Bakin, A.; Behrends, A.; Wagner, A.; Waag, A. Fabrication of ZnO Nanostructures. In Zinc Oxide Nanostructures; Pan Stanford Publishing Pte Ltd.: Singapore, 2014; ISBN 978-981-4411-33-2.

2. Liu, S.Y.; Chen, T.; Wan, J.; Ru, G.P.; Li, B.Z.; Qu, X.P. The effect of pre-annealing of sputtered ZnO seed layers on growth of $\mathrm{ZnO}$ nanorods through a hydrothermal method. Appl. Phys. A 2009, 94, 775780.

3. Xu, J.; Bertke, M.; Li, X.; Mu, H.; Zhou, H.; Yu, F.; Hamdana, G.; Schmidt, A.; Bremers, H.; Peiner, E. Fabrication of $\mathrm{ZnO}$ nanorods and Chitosan@ZnO nanorods on MEMS piezoresistive self-actuating silicon microcantilever for humidity sensing. Sens. Actuators B Chem. 2018, 273, 276-287. 
4. Xu, J.; Bertke, M.; Gad, A.; Yu, F.; Hamdana, G.; Bakin, A.; Peiner, E. Fabrication of ZnOnanorods on MEMS piezoresistive silicon microcantilevers for environmental monitoring. Proceedings 2017, 1, 290.

5. Huang, J.S.; Lin, C.F. Influences of $\mathrm{ZnO}$ sol-gel thin film characteristics on $\mathrm{ZnO}$ nanowire arrays prepared at low temperature using all solution-based processing. J. Appl. Phys. 2008, 103, 014304.

6. Xu, J.; Bertke, M.; Li, X.; Mu, H.; Peiner, E. Self-actuating and self-sensing ZNO nanorods/chitosan coated piezoresistive silicon microcantilever for humidity sensing. In Proceedings of the 2008 IEEE Micro Electro Mechanical Systems (MEMS), Belfast, UK, 2018; pp. 206-209.

(C) 2018 by the authors; Licensee MDPI, Basel, Switzerland. This article is an open access article distributed under the terms and conditions of the Creative Commons Attribution (CC BY) license (http://creativecommons.org/licenses/by/4.0/). 